\title{
HISTORICAL RECORDS OF ZZ CETI VARIABLES
}

Martha H. Liller

Harvard College Observatory

James E. Hesser

Dominion Astrophysical Observatory

As a consequence of the discovery of the high-frequency variations that define the $\mathrm{ZZ}$ CetI class of varlables, observations of them or of new candidates have naturally tended to sample preferentially the period range from 1 or 2 seconds to $~ 30$ minutes in unfiltered 11ght. Hence, 1ittle is known about varlability on time scales > 30 minutes, with the possible exception of some curlosities in the high-frequency literature and in Eggen's monumental photoelectric UBV observations; a brief summary of some potentially disquieting cases has been given recently by Hesser, Lasker and Neupert (1979). Inasmuch as the extstence of unexplained observational discrepancies st11l admit the possibility that the high-frequency varlations may be but one manifestation of the type or time scales of white-dwarf varlability, we have measured several $\mathrm{ZZ}$ Cet 1 varlables by Iris photometry and by visual estimates on the plates of the Harvard collection. Based on an average of 50 observations per star over 55 years, going back to the $1890^{\prime} \mathrm{s}$, we find no positive indications of variations in excess of $\pm 0.10-0.15 \mathrm{mag}$ for any of the following stars:
BPM 30551
GD 99
G $180-23$
R 548
G 117 - B 15a
G $169-34$
BPM 31594
G $44-32$
G 207-09
HL TAU -76
GD 154
LFT 1679
G $38-29$
L $19-2$
G $29-38$

In spite of the 11mitations on precision imposed by the plate material, the results provide some assurance that the $\mathrm{ZZ}$ Cet1 stars do not suffer large, very low-frequency amplitude modulations.

\section{REFERENCE}

Hesser, J.E., Lasker, B.M., and Neupert, H.E., 1979, Ap.J. Supp1. (in press). 